\title{
Synergy of Monopolar Radiofrequency Heating and Targeted Pressure Energy as an Innovative Approach to Cellulite Reduction
}

\author{
Diane Duncan*, M.D., FACS \\ Plastic Surgical Associates, Fort Collins CO, USA
}

*Corresponding author: Diane Duncan, M.D., FACS, Plastic Surgical Associates, Fort Collins CO, 1701 East Prospect Road, USA.

To Cite This Article: Diane Duncan, M.D., FACS, Synergy of Monopolar Radiofrequency Heating and Targeted Pressure Energy as an Innovative Approach to Cellulite Treatment. Am J Biomed Sci \& Res. 2021 - 12(1). AJBSR.MS.ID.001717. DOI: 10.34297/AJBSR.2021.12.001717.

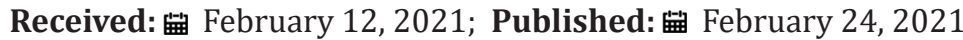

\begin{abstract}
As much as $90 \%$ of women are experiencing altered skin topography due to cellulite. Nowadays, various treatment modalities are utilized for cellulite treatment, focusing on different approaches to address such a multifactorial condition. This article describes a unique technical solution combining two proven technologies in a standalone device to treat all major factors contributing to cellulite. The device utilizes a single applicator system that simultaneously delivers mechanical and thermal energy resulting in structural and functional changes in dermis and hypodermis.
\end{abstract}

Keywords: Cellulite; Radiofrequency; Targeted Pressure Energy

Abbreviations: AAD: American Academy of Dermatology; RF: Radio Frequency; TPE: Targeted Pressure Energy

\section{Introduction}

Gynoid lipodystrophy, commonly known as cellulite manifests as an alteration of the skin topography that occurs mainly in women on the pelvic region, lower limbs and abdomen. It is characterized by a padded or orange peel's appearance with dimpling and nodularity caused by herniation (protrusion) of subcutaneous fat within fibrous connective tissue [1]. Cellulite affects over $90 \%$ of women nearly in all stages of their lives, with the most noticeable onset in the age of 20-30 years. A variety of invasive and noninvasive treatment options are available to improve the appearance of cellulite. American Academy of Dermatology (AAD) recognizes various modalities that can effectively treat cellulite-e.g., thermal and mechanical energy, lasers and submission. Given the risks, downtime and inconvenience often related to minimally invasive or invasive approaches, non-invasive treatments are preferably sought by the patients.

Radiofrequency (RF) heating and mechanical energy are the gold standards in non-invasive cellulite treatment, since they can induce structural changes in the dermis and subcutaneous tissue to target multiple causes of cellulite effectively. There are currently various devices on the market that use either RF or mechanical energy to treat cellulite in a standalone regime or consecutively to enhance the results. Although it was evidenced that consecutive treatments lead to higher efficiency via the synergy of various physiological effects induced by the respective technologies [24], the simultaneous application may achieve even superior results due to the immediate synergy. Nevertheless, the technical requirements to combine such distinct technologies only allowed for their consecutive use so far. Recently, however, the latest advancements in technology solutions have emerged, and for the first-time simultaneous therapy with RF and mechanical energy has become available for cellulite.

Technical Solution Allowing Simultaneous
Application

Emtone (BTL Industries Inc, Boston, MA) is the first and only device that simultaneously combines radiofrequency and mechanical targeted pressure energy (TPE) for the treatment of cellulite in the single therapy due to a unique technical solution developed and patented by BTL Industries Inc. Both energies 
are generated in the main device and effectively delivered to the desired area affected by cellulite using a handpiece applicator, equipped by easy to manipulate capacitive sense buttons and display which indicates the basic parameters of the therapy (Figure 1). The simultaneous application allows for the synergistic effect on fat protrusions and disruption of damaged collagen fibers followed by neocollagenesis and neoelastogenesis. This leads to thickening of the dermis, a better interlobular septage organization in the hypodermis, and improved skin appearance [4-6] (Figure 1).

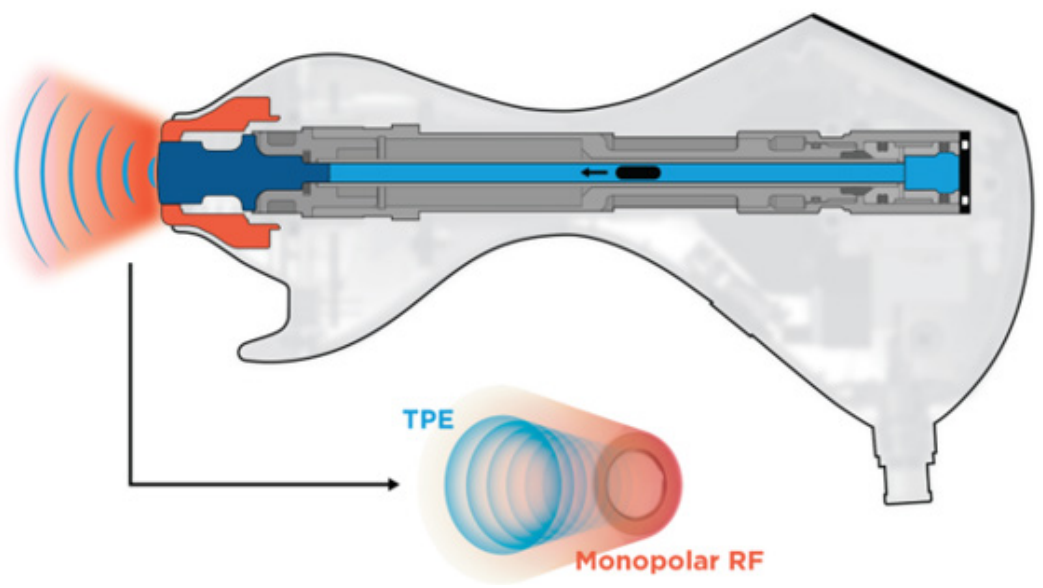

Figure 1: Emtone device simultaneously combines targeted pressure energy (TPE) and monopolar radiofrequency (RF) in a single handpiece.

\section{Radiofrequency Component}

Radio frequency refers to electromagnetic waves in the frequency range of roughly $20 \mathrm{kHz}$ to $300 \mathrm{GHz}$. These are the frequencies with the ability to create heat through the oscillation of molecules while propagating within the tissue. Oscillating electrical currents force collisions between charged molecules and ions leading to friction and the formation of thermal energy. The heating effects of the RF technology can be used for a wide range of aesthetic applications such as for cellulite treatment $[4,7-$ 9]. Today, it is well known that RF heating of the skin leads to a thermally mediated reaction in the dermis associated with collagen remodeling followed by tissue tightening. Furthermore, the heat delivered beyond the dermis to the subcutaneous layer is presumed to be absorbed by adipocytes and interlobular septa to supposedly induce adipocyte shrinkage and better septa organization [4]. Subsequently, these processes result in the improvement of various tissue characteristics $[2,4]$.

Emtone utilizes monopolar RF (with a frequency of $447 \mathrm{kHz}$ ) that is optimal for deeper penetration. The RF energy is emitted by a solid metal electrode, which is in direct contact with the patient's skin. The generated currents travel to the grounding pad ensuring a safe flow of the RF energy through the treated area (see Figure 2) and controlled energy delivery (Figure 2).

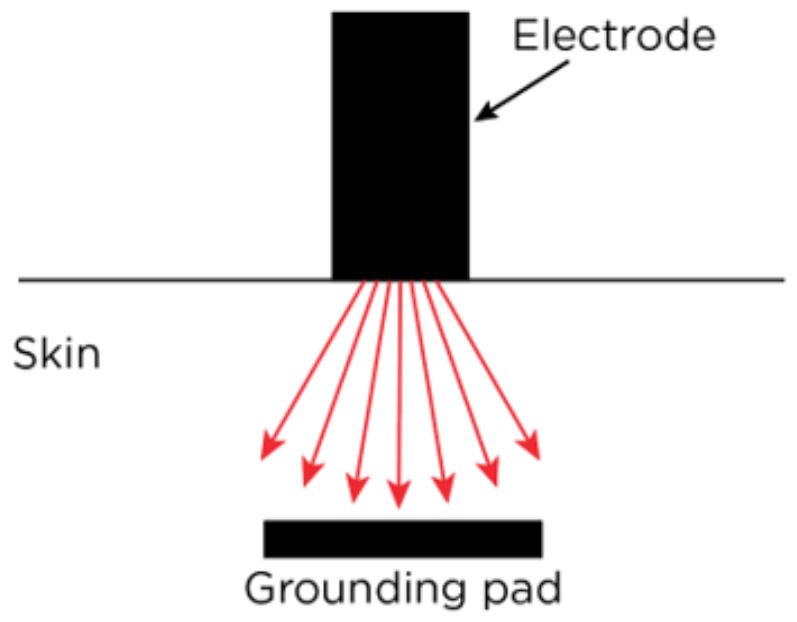

Figure 2: Energy flow during monopolar RF application, RF currents are schematically illustrated by the red lines. 


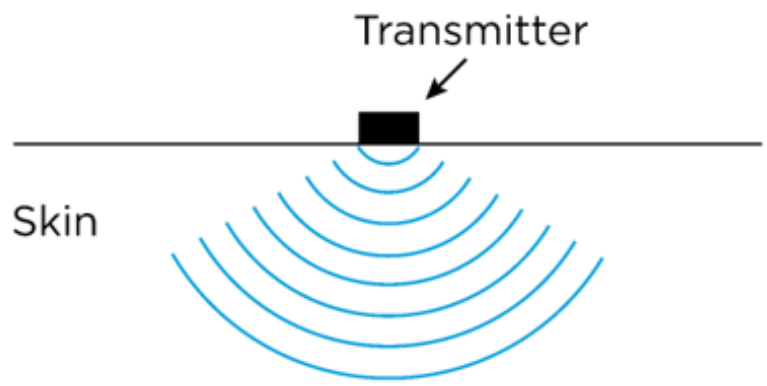

Figure 3: Blue lines illustrate transmission of the TPE energy into living tissue, propagation of the pressure waves.

\section{Targeted Pressure Energy Component}

Besides enhancing RF-induced changes, it is also observed that mechanical stimulation may improve local blood circulation and promote neovascularization [4]. Pressure waves should further increase cell proliferation of collagen and elastin fibers to improve skin elasticity and to revitalize the dermis. TPE also positively affects lymph transport, supposedly another key aspect associated with cellulite. TPE mechanical stimulation is based on the ballistic principle. The terminal apparatus of the TPE component is embedded in the handpiece and consists of a tube with a floating projectile accelerated towards an applicator tip by the pneumatic system. The compressor of the pneumatic system is placed in the main unit and in combination with mechanical parts it transforms TPE energy to the target tissue. A projectile is moved by the compressed air, while hitting a transmitter that conveys energy from the impact to the patient's body. This action is repeated in quick succession (frequency of $10 \mathrm{~Hz}$ ) while resulting in the pressure waves of significant intensities (Figure $1 \& 3$ ).

\section{Technical Challenges}

The greatest challenge with developing such a device was combining two different technologies in a single applicator while maintaining high quality and safety standards. The applicator does not contain any visible fitting (screws, holes etc.). One of the most important sub-challenges was developing a system that can transmit RF energy through mechanically movable parts of the applicator while keeping the warranty of those components up to $1,000,000$ shots. At the same time, with patients' comfort in mind, technical solutions possess impedance intelligence and real-time temperature monitoring for safe but highly effective treatment.

\section{Conclusion}

EMTONE is the only device of its kind that allows the use of a simultaneous combination of two proven technologies in cellulite treatment. This patented concept combining targeted pressure energy with radiofrequency heating allows leveraging both clinical and commercial synergies of combination from a single device applicator.

\section{References}

1. Rossi Ana \& Vergnanini Andre (2000) Cellulite: A review. Journal of the European Academy of Dermatology and Venereology : JEADV 14: 25162.

2. Luebberding Stefanie \& Krueger Nils, Sadick Neil (2015) Cellulite: An Evidence-Based Review. American journal of clinical dermatology 16(4): 243-256.

3. Cellulite treatments: What really works? American Academy of Dermatology Association.

4. Kinney BM, Kanakov D, Yonkova P (2020) Histological examination of skin tissue in the porcine animal model after simultaneous and consecutive application of monopolar radiofrequency and targeted pressure energy. Journal of Cosmetic Dermatology 19: 93-101.

5. Fritz K, Salavastru C, Gyurova M (2018) Clinical evaluation of simultaneously applied monopolar radiofrequency and targeted pressure energy as a new method for noninvasive treatment of cellulite in postpubertal women. J Cosmet Dermatol 17: 36-364.

6. Fritz K, Salavastru C, Gyurova M (2018) Reduction of abdominal skin laxity in women postvaginal delivery using the synergistic emission of radiofrequency and targeted pressure energies. J Cosmet Dermatol 17(5): 766-769.

7. Angélica Rodrigues de Araújo, Viviane Pinheiro Campos Soares, Fernanda Souza da Silva, Tatiane da Silva Moreira (2015) Radiofrequency for the treatment of skin laxity: myth or truth. Anais Brasileiros de Dermatologia 90(5): 707-721.

8. Alizadeh Z, Halabchi F, Mazaheri R, Abolhasani M, Tabesh M, et al. (2016) Review of the Mechanisms and Effects of Noninvasive Body Contouring Devices on Cellulite and Subcutaneous Fat. Int J Endocrinol Metab 14(4): e36727.

9. Emilia del Pino M, Rosado RH, Azuela A, Graciela Guzmán M, Argüelles D, et al. (2006) Effect of controlled volumetric tissue heating with radiofrequency on cellulite and the subcutaneous tissue of the buttocks and thighs. J Drugs Dermatol 5(8): 714-22. 